\title{
Rat Ultrasonic Vocalization Shows Features of a Modular Behavior
}

\author{
Tobias Riede \\ Department of Biology and National Center for Voice and Speech, University of Utah, Salt Lake City, Utah 84112
}

Small units of production, or modules, can be effective building blocks of more complex motor behaviors. Recording underlying movements of vocal production in awake and spontaneously behaving male Sprague Dawley rats interacting with a female, I tested whether the underlying movements of ultrasonic calls can be described by modules. Movements were quantified by laryngeal muscle EMG activity and subglottal pressure changes. A module was defined by uniformity in both larynx movement and pressure pattern that resulted in a specific spectrographic feature. Modules are produced either singly (single module call type) or in combination with a different module (composite call type). Distinct modules were shown to be linearly (re)combined. Additionally, I found that modules produced during the same expiratory phase can be linked with or without a pause in laryngeal activity, the latter creating the spectrographic appearance of two separate calls. Results suggest that combining discrete modules facilitates generation of higher-order patterns, thereby increasing overall complexity of the vocal repertoire. With additional study, modularity and flexible laryngeal-respiratory coordination may prove to be a basal feature of mammalian vocal motor control.

Key words: breathing; larynx; motor control

\section{Introduction}

A question in the field of motor control is how the CNS generates muscle activity patterns necessary to produce different behaviors. Recombining small units of production is an effective mechanism to increase complexity in a system with limited computational capacity (Bizzi et al., 2008). In the case of vocal behavior, neural circuits generating automatic rhythms (e.g., Jürgens and Hage, 2007) underlie the generation of different vocal patterns via fine-tuned movements. Spectrotemporal analysis suggests that calls in nonhuman mammals consist of discrete syllables emitted alone or in combination (e.g., Robinson, 1984), implying that a mammalian vocal repertoire consisting of a high number of different vocal patterns relies on a smaller number of motor programs, which are differently (re)combined. Alternatively, each call type could be facilitated by its own motor program. Unfortunately, the underlying movements of vocalizations cannot be inferred unambiguously from spectrograms because active and passive components of vocal production demonstrate nonlinear characteristics (e.g., Kobayasi et al., 2012). However, one way to determine the existence of small building blocks is to characterize and compare muscle activity patterns between different vocal

\footnotetext{
Received Jan. 20, 2014; revised April 2, 2014; accepted April 13, 2014.

Author contributions: T.R. designed research; T.R. performed research; T.R. contributed unpublished reagents/ analytic tools; T.R. analyzed data; T.R. wrote the paper.

This work was supported by National Institutes of Health Grant R01-DC-006876, University of Utah Study Design and Biostatistics Center, with funding in part from the National Center for Research Resources and the National Center for Advancing Translational Sciences, and National Institutes of Health Grant 8UL1TR000105 (formerly UL1RR025764).

The authors declare no competing financial interests.

Correspondence should be addressed to Dr. Tobias Riede, Department of Biology, University of Utah, 257 South 1400 East, Salt Lake City, UT 84112. E-mail: t.riede@utah.edu.

Copyright $\odot 2014$ the authors $\quad 0270-6474 / 14 / 346874-05 \$ 15.00 / 0$
}

behaviors and/or describe the kinematics of generated motor patterns. Hereafter I use the term "module" to refer to a uniform motor activity associated with an acoustic utterance.

Mammalian vocalization requires coordinating the movements of larynx, vocal tract, and respiratory system (e.g., Jürgens, 2002). Both vocal fold posturing by intrinsic laryngeal muscles and breathing movements that build up lung pressure required to generate an air flow are critical for normal sound production. This includes ultrasonic vocalizations (USVs) in rats (Riede, 2011). Rats have a particularly rich repertoire of ultrasonic calls (e.g., Wright et al., 2010) that feature prominently in intraspecific communication (e.g., Wöhr and Schwarting, 2012). The stereotypy of underlying motor patterns has been demonstrated only at the call level (Riede, 2013) because studies in other mammals suggested that the "call" is the smallest vocal unit (e.g., Miller et al., 2003). Generation of some calls seems to involve more than one motor pattern (Riede, 2013), suggesting that smaller units exist below the call level. Motor patterns should be similar between single module calls (produced by a single module) and composite calls (produced by multiple modules) if one and the same motor program is recruited to produce both call types. Two hypotheses were addressed: (1) whether motor patterns of a single module call are identical to those used in composite calls; and (2) whether rats (re)combine these modules to generate composite calls. The first hypothesis was tested by comparison of subglottal pressure and EMG laryngeal muscle activities in single module and composite calls. The second hypothesis was tested by determining modular characteristics of composite call types and their rates. Testing these two hypotheses also permitted the detection of incompletely assembled modules, which was unexpected for USVs in rats. The associated laryngeal-respiratory coordination not only provided further support for a modular characteristic of 

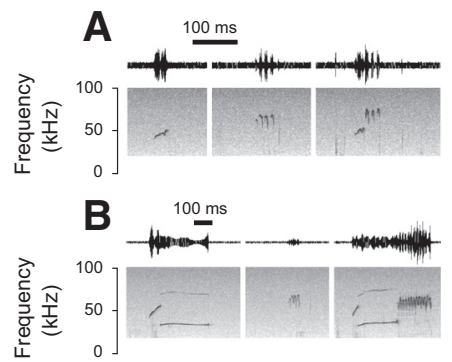

章衣
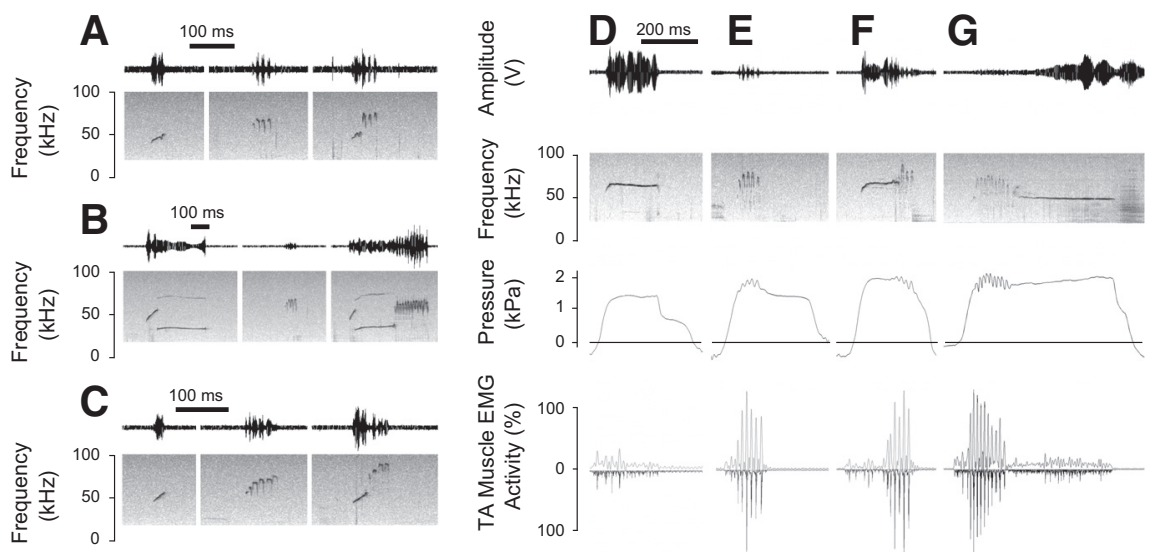

Figure 1. Rats produce different call types in the $50 \mathrm{kHz}$ call category, for example, complex calls (first call in $A$ ), split calls (first call in $\boldsymbol{B}$ ), upward calls (first call in $\boldsymbol{C}$, flat calls $(\boldsymbol{D})$, trills $(\boldsymbol{E}$, and second calls in $\boldsymbol{A}-\boldsymbol{C}$ ). The complex call is characterized by several small fundamental frequency inversions in contrast to the upward call (Wright et al., 2010). Complex calls, split calls, upward calls, flat calls, and trills are considered single-module calls because the underlying motor patterns are uniform and call type specific. Complex, split, upward, and flat modules are also produced in association with trills, as complex-trill composite calls (third call in $\boldsymbol{A}$ ), split-trill composite calls (third call in $\boldsymbol{B}$ ), upward-trill composite calls (third call in $\boldsymbol{C}$, flat-trill $(\boldsymbol{F})$, or trill-flat composite calls $(\boldsymbol{G})$. Sound is depicted as time waveform (top; relative change in output voltage of microphone signal) and spectrographically (second panel; bandpass filtered 20-100 kHz). D-G, Subglottal pressure (third panel, horizontal line indicates ambient pressure) and thyroarytenoid muscle EMG activity (fourth panel). EMG activity is displayed as a rectified original trace (downward) and low-pass filtered ( $200 \mathrm{~Hz}$ ) trace (upward). EMG activity is expressed relative to maximum activity measured during swallowing.

underlying motor patterns of USVs but demonstrated a key feature of laryngeal-respiratory coordination shared with human speech production.

\section{Materials and Methods}

Study animals and behavioral paradigm. I investigated the vocal behavior of male Sprague Dawley rats (Institutional Animal Care and Use Committee protocol 12-05006, University of Utah), while they were permitted to interact with females. Each male was housed in a standard rodent cage, and a familiar female was placed in an adjacent cage connected via a short 2 -cm-wide tube through which the animals could have physical contact. The male was allowed contact with the female through the tube once per day during a $5 \mathrm{~min}$ recording session. A microphone (AvisoftBioacoustics, CM16/CMPA-5V) was placed $10 \mathrm{~cm}$ above the male's cage. Sound was recorded with Avisoft Recorder software using a NIDAQ 6212 acquisition device ( $200 \mathrm{kHz}$ sampling rate). The female's cage was soundshielded as a measure against male-female overlap of recorded vocalizations. Sound recordings were made in 10 males, and eight of these subsequently underwent implant surgery to record both muscle activity and subglottal pressure.

EMG and subglottal pressure recordings. Subglottal pressure was measured by implanting a stainless steel tube in the upper third of the trachea and connecting it to a pressure transducer (model FHM-02PGR-02; Fujikura) housed in a backpack. EMG activity of one intrinsic laryngeal muscle (thyroarytenoid muscle) was recorded with bipolar silver electrodes. EMG recordings were differentially amplified (model EX4-400, Dagan Corporation), bandpass filtered $(100-3000 \mathrm{~Hz})$, full-wave rectified, low-pass filtered $(200 \mathrm{~Hz})$, and normalized to a rat's maximum EMG activity recorded during swallowing (using PRAAT software; www. praat.org). The calibrated pressure signal and EMG activity were recorded simultaneously with the sound signal (Riede, 2011, 2013).

Analysis. Five call types were characterized (following Wright et al., 2010): complex (two or more fundamental frequency inversions), split (one or two sudden jumps in fundamental frequency), upward (fundamental frequency increases by $>5 \mathrm{kHz} / 10 \mathrm{~ms}$ ), flat (fundamental frequency changes $<5 \mathrm{kHz} / 10 \mathrm{~ms}$ ), and trill (three or more fast up-and-down frequency modulations). The associated motor patterns were analyzed in single module calls and in five composite calls defined as follows: flat-trill, trill-flat, split-trill, complex-trill, and upward-trill.
To address the hypothesis of modular similarity between single module and composite calls, mean subglottal pressure, mean EMG activity, and the coefficient of variation $(\mathrm{CV})$ of EMG activity were compared across all call types. Additionally, EMG bursting rate, EMG burst amplitude, number of EMG bursts, and EMG burst duration were measured in trills and complex calls because EMG activity in both call types is characterized by a regular bursting pattern (Riede, 2013). Similarity between single modules and modules in composite calls was compared using mixed effect linear regression with treatment condition as the fixed effect predictor (single module vs module in composite call) and the individual rat as a random effect. This mimicked a paired $t$ test while accounting for lack of independence in conducting multiple observations per rat. A false discovery rate adjustment (BenjaminiHochberg procedure; Benjamini and Hochberg, 1995) was performed to keep the false discovery rate at the nominal $\alpha 0.05$ level. Data were analyzed using Stata-13 statistical software (StataCorp).

The frequency of both occurrence of composite calls in general and various composite call types was determined to investigate the way in which rats assemble modules. Examples of incompletely assembled modules are also described.

\section{Results}

Spectrotemporal features (flat, split, upward, complex) were produced as separate calls or in combination with trills to form composite calls (Fig. 1). The cumulative rate of composite calls ranged between $0.1 \%$ and $17 \%$ (mean, $3.8 \%$ ) in 10 male rats. Composite calls were only produced if vocal activity exceeded a threshold of 80 calls per 5 minute recording period. The correlation between an individual's total number of calls (per 5 minute session) and composite call rate was significant $\left(r^{2}=0.66 ; t=4.1, p<0.01\right)$ beyond this threshold.

Flat-trill composite calls (Fig. $1 F$ ) were the most frequently produced composite call type (in 8 rats, $45 \pm 25 \%$ of all composite calls, mean $\pm \mathrm{SD})$, followed by complex-trill composite calls (Fig. 1A) (in 7 rats, $31 \pm 27 \%$ ), split-trill composite calls (Fig. 1B) (in 6 rats, $17 \pm 11 \%$ ), and upward-trill composite calls (Fig. 1C) (in 2 rats, $10 \%$ and $1 \%$, respectively). Other composite call types included trill-flat composite calls (Fig. $1 G$ ) (in 4 rats, $7 \pm 4 \%$ ) and calls comprised of three or more of the above-mentioned features $(8$ rats, $<1 \%)$.

Underlying motor patterns were quantified using variables derived from laryngeal muscle EMG activity and mean subglottal pressure. Mean subglottal pressure, mean and CV EMG were not different for flat, split, and upward calls and their respective elements in composite calls (Table 1).

Mean subglottal pressure and EMG variables revealed several differences between complex calls/trills and their respective elements in composite calls. Mean EMG activity, EMG burst duration, EMG burst amplitude, number of bursts, and mean subglottal pressure did not differ between complex calls and complex call elements in composite calls, but EMG CV and EMG burst rate were marginally smaller in single module calls (Table 1). For trill calls and trill call elements, no significant differences were found in mean EMG activity, EMG CV, EMG burst amplitude, and EMG burst rate, but fewer bursts were produced in composite calls (Table 1) and both burst duration and mean 
Table 1. Mean \pm SE of subglottal pressure and laryngeal EMG muscle activity variables measured in five modules in single module (SMC) and composite calls (CC) demonstrated few differences (mixed effect linear regression) ${ }^{a}$

\begin{tabular}{|c|c|c|c|c|c|}
\hline & Flat $N=(8,309)$ & Split $N=(5,117)$ & Upward $N=(1,33)$ & Complex $N=(5,143)$ & Trill $N=(8,454)$ \\
\hline \multicolumn{6}{|c|}{ Ps mean (kPa) } \\
\hline SMC & $0.87 \pm 0.09$ & $0.87 \pm 0.16$ & $0.62 \pm 0.01$ & $0.93 \pm 0.10$ & $0.94 \pm 0.10$ \\
\hline \multirow[t]{2}{*}{$\mathrm{CC}$} & $0.88 \pm 0.09$ & $0.88 \pm 0.16$ & $0.64 \pm 0.01$ & $0.93 \pm 0.10$ & $1.05 \pm 0.11$ \\
\hline & $z=0.6, p=0.61$ & $\mathrm{z}=0.4, p=0.72$ & & $z=0.2, p=0.85$ & $z=4.34, p=0$ \\
\hline \multicolumn{6}{|c|}{ EMG mean (mV) } \\
\hline SMC & $15.6 \pm 2.3$ & $19.4 \pm 3.3$ & $22.9 \pm 0.9$ & $34.6 \pm 6.0$ & $57.1 \pm 5.5$ \\
\hline \multirow[t]{2}{*}{$C \mathrm{C}$} & $16.7 \pm 2.3$ & $20.7 \pm 3.3$ & $25.4 \pm 1.1$ & $37.2 \pm 5.9$ & $57.9 \pm 5.5$ \\
\hline & $z=2.35, p=0.06$ & $z=1.49, p=0.21$ & & $z=1.54, p=0.24$ & $z=0.74, p=0.54$ \\
\hline \multicolumn{6}{|l|}{ EMG CV } \\
\hline SMC & $1.09 \pm 0.05$ & $1.06 \pm 0.06$ & $1.14 \pm 0.03$ & $1.51 \pm 0.18$ & $1.49 \pm 0.07$ \\
\hline \multirow[t]{2}{*}{$\mathrm{CC}$} & $1.11 \pm 0.05$ & $1.09 \pm 0.06$ & $1.13 \pm 0.03$ & $1.62 \pm 0.18$ & $1.48 \pm 0.07$ \\
\hline & $z=1.51, p=0.22$ & $z=1.51, p=0.22$ & & $z=2.56, p=0.04$ & $z=0.98, p=0.43$ \\
\hline \multicolumn{6}{|c|}{ EMG burst rate $(\mathrm{Hz})$} \\
\hline SMC & NA & NA & NA & $96.1 \pm 3.1$ & $91.7 \pm 0.76$ \\
\hline \multirow[t]{2}{*}{$\mathrm{CC}$} & & & & $106.7 \pm 3.0$ & $90.5 \pm 0.76$ \\
\hline & & & & $z=5.93, p=0$ & $z=0.77, p=0.558$ \\
\hline \multicolumn{6}{|c|}{ EMG burst duration (ms) } \\
\hline SMC & NA & NA & NA & $1.57 \pm 0.14$ & $1.78 \pm 0.06$ \\
\hline \multirow[t]{2}{*}{$C C$} & & & & $1.69 \pm 0.14$ & $1.88 \pm 0.06$ \\
\hline & & & & $z=2.04, p=0.09$ & $z=3.31, p=0.005$ \\
\hline \multicolumn{6}{|c|}{ EMG burst amplitude (mV) } \\
\hline SMC & NA & NA & NA & $130 \pm 1.6$ & $232 \pm 2.5$ \\
\hline \multirow[t]{2}{*}{$\mathrm{CC}$} & & & & $148 \pm 1.6$ & $226 \pm 5.9$ \\
\hline & & & & $z=2.35, p=0.053$ & $z=1.3, p=0.28$ \\
\hline \multicolumn{6}{|c|}{ No. of EMG bursts } \\
\hline SMC & NA & NA & NA & $3.76 \pm 0.26$ & $7.9 \pm 0.48$ \\
\hline \multirow[t]{2}{*}{$C \mathrm{C}$} & & & & $3.34 \pm 0.25$ & $6.63 \pm 0.45$ \\
\hline & & & & $z=2.31, p=0.053$ & $z=3.85, p=0$ \\
\hline
\end{tabular}

${ }^{a}$ Variables were mean subglottal pressure (Ps mean), mean thyroarytenoid muscle EMG activity (EMG mean), CV of EMG activity (EMG CV), EMG burst rate, EMG burst duration, peak EMG burst amplitude, and total number of EMG trill bursts. From each rat and call type, $14-20$ calls were analyzed. N indicates the number of rats and the total number of calls; NA, not applicable. No statistical test was performed for upward calls because data from only one rat were available.

subglottal pressure were smaller in single module calls. Because each EMG burst is associated with one frequency modulation in a trill (Riede, 2013), this means that trill elements in a composite call were shorter than single module trills.

One particular feature does suggest flexible assemblage of modules. Figure 2 shows examples of modules produced during the same expiratory phase that are separated by interruptions in laryngeal EMG activity lasting $30-90 \mathrm{~ms}(n=3$ rats): Figure $2 A$, a flat and a trill module; Figure $2 B$, a composite call and a trill; and Figure $2 C$, a flat, a trill, and another flat module appear incompletely assembled. Between $0.4 \%$ and $2 \%$ of all composite calls in 7 of the 8 rats demonstrated such pauses, which ranged in duration between 14 and $342 \mathrm{~ms}$ (Table 2). The preceding inspirations were significantly longer (paired $t$ test, $t=3.9, p<0.01$ ) and deeper (paired $t$ test, $t=5.5, p<0.01$ ) than those before composite calls without pause (Table 2).

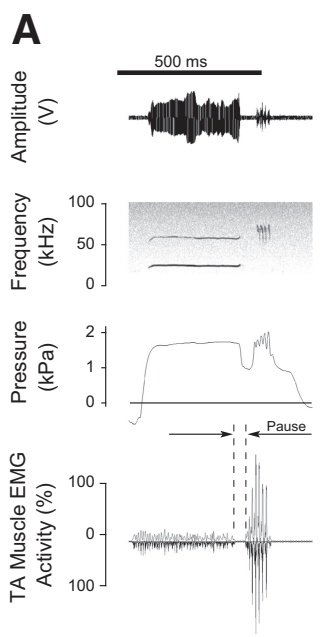

B
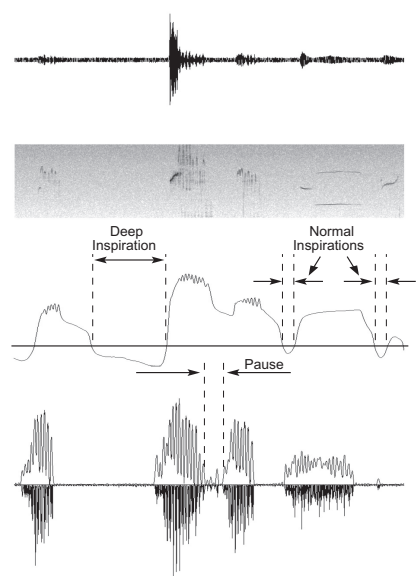

Figure 2. Three examples of composite calls in which two modules are produced during the same expiratory phase. The interruption of the laryngeal muscle EMG activity and the separation of associated spectrotemporal features create the appearance of separate calls in the spectrogram. $\boldsymbol{A}$, A flat and a trill are separated by a $20 \mathrm{~ms}$ pause. $\boldsymbol{B}, \mathrm{A}$ complex-trill and a trill single module are separated by a 50 ms pause. The utterance is preceded by a trill call and followed by a split call. The recording shows the magnitude of a large deep inspiration compared with two normal inspirations. C, A flat, a trill, and another flat are separated by two $20 \mathrm{~ms}$ pause. The pressure signal before the onset of the incomplete composite call reaches greater negative pressure, suggesting deep inspiration collecting a larger air volume in the lungs available during the next expiratory phase, thereby facilitating several separate modules during a single breath.

\section{Discussion}

The results presented here lend support to the hypothesis that rats share three important features of a modular vocal behavior: (1) linear combination of modules, (2) module recombination, and (3) flexible laryngeal-respiratory coordination.
Modular organization of behavior predicts that, if the same motor program is recruited for both single module and composite behaviors, the resulting motor patterns should be similar. Because observed motor patterns for flat, split, and upward calls and their respective call elements in composite calls are similar, each 
Table 2. Average duration of pauses between two modules generated during a single expiration and inspirations preceding composite calls ${ }^{a}$

\begin{tabular}{|c|c|c|c|c|c|}
\hline \multirow[b]{2}{*}{ Rat } & \multirow[b]{2}{*}{ Pause (ms) } & \multicolumn{2}{|c|}{ Preceding inspiration "A" } & \multicolumn{2}{|c|}{ Preceding inspiration " $\mathrm{B}$ " } \\
\hline & & Duration (ms) & Pressure (kPa) & Duration (ms) & Pressure $(\mathrm{kPa})$ \\
\hline 70 & $45 \pm 28(N=48)$ & $208 \pm 70$ & $-1.0 \pm 0.6$ & $115 \pm 54(N=20)$ & $-0.7 \pm 0.2$ \\
\hline 69 & $88 \pm 92(N=22)$ & $181 \pm 97$ & $-0.8 \pm 0.3$ & $91 \pm 24(N=20)$ & $-0.7 \pm 0.1$ \\
\hline 67 & $88 \pm 94(N=10)$ & $146 \pm 67$ & $-1.1 \pm 0.9$ & $88 \pm 30(N=20)$ & $-0.7 \pm 0.5$ \\
\hline 63 & $81 \pm 42(N=3)$ & $209 \pm 102$ & $-0.9 \pm 0.8$ & $86 \pm 29(N=20)$ & $-0.5 \pm 0.1$ \\
\hline 62 & $67 \pm 71(N=26)$ & $243 \pm 109$ & $-1.1 \pm 0.4$ & $115 \pm 60(N=20)$ & $-0.5 \pm 0.2$ \\
\hline 56 & $59 \pm 16(N=2)$ & $162 \pm 131$ & $0.5 \pm 0.2$ & $66 \pm 9(N=20)$ & $0.3 \pm 0.1$ \\
\hline 41 & $76 \pm 33(N=9)$ & $181 \pm 98$ & $-0.4 \pm 0.1$ & $80 \pm 53(N=16)$ & $-0.2 \pm 0.1$ \\
\hline 39 & $21(N=1)$ & 401 & -0.7 & $42 \pm 13(N=20)$ & $-0.4 \pm 0.1$ \\
\hline
\end{tabular}

${ }^{a}$ Rats produced composite calls in which multiple modules were produced during the same expiration (preceded by inspiration " $\mathrm{A}$ ") but were separated by a pause of laryngeal muscle EMG activity. $N$ indicates the number of incompletely assembled composite calls found. Inspirations " $\mathrm{A}$ " were longer (duration) and deeper (pressure) than before uninterrupted composite calls (inspiration " $\mathrm{B}$ "). Inspirations before 20 utterances were averaged (mean $\pm \mathrm{SD}$ ).

pair could have conceivably been generated by the same individual motor program. The demonstrated differences between variables for complex and trill calls and call elements, respectively, may point to employment of different motor programs. However, because the differences were very small (Table 1), this could simply reflect coarticulation effects, i.e., the fine structure of the underlying movements could be influenced largely by the movement immediately preceding or following it (Flash and Hochner, 2005). For example, the shorter average duration of trill modules contained in composite calls likely reflects a respiratory constraint on overall call duration: if trill modules are produced in close association with another module, they cannot be as long as they might be when uttered singly.

Modules ("gestures," in human and songbird research; e.g., Amador et al., 2013) have also been suggested as basis of human speech. Speech movements produce a time-dependent acoustic signal with spectrotemporal features that are determined by quantifiable variables (e.g., amount of vocal fold tension, activity of laryngeal and vocal tract muscles, aerodynamic variables). Individual speech sounds ("phonemes" or "syllables") are (re)combined to form words (e.g., MacNeilage, 1970; Browman and Goldstein, 1989; Kent et al., 2000). Derived from this concept are theories of how underlying movements are controlled. For example, one popular theory assumes that a speaker's objective is to produce an intelligible acoustic pattern requiring timedependent patterns of auditory and somatosensory feedback (e.g., Perkell, 2012; Hickok, 2012). Obviously, there are many differences between human speech and rat USVs, but the newly developed rat preparation may prove an informative model for determining how vocal motor control evolved in mammals. Whereas auditory feedback, to date, seems to play no meaningful role in rodent vocal production (Hammerschmidt et al., 2012; Kikusui et al., 2011; Mahrt et al., 2013; but see Arriaga et al., 2012, for effects in surgically deafened mice), somatosensory feedback mechanisms remain poorly understood (e.g., Smotherman, 2007). The mechanism of module (re)combination likely requires an increased capacity for vocal-respiratory integration, regardless of either historical contingency or feedback/feedforward dependency, because the coordination of larynx and respiratory system is what produces smooth, temporally ordered vocal sequences in rats. Greater song complexity in songbirds is also associated with larger song control system anatomy (e.g., Brenowitz and Arnold, 1986; Moore et al., 2011). Pinpointing the neurological candidates for control circuitry requires a functionally divergent outgroup; an obvious comparison for rats is mice. The large vocal repertoire of mice appears to contain only single module calls (e.g., Holy and Guo, 2005; Scattoni et al., 2008). Thus, any neural correlates for module assembly should be present in rats but not in mice.
Surprisingly, two or more separate laryngeal motor patterns were produced during the same expiration in 0.4 to $2 \%$ of composite calls. This observation has three implications. First, this laryngeal maneuver creates the spectrographic appearance of two calls, pointing to limitations in inferring motor control patterns solely from spectrographic information. Second, it further suggests modular characteristics of rat USV, given that variable recruitment of laryngeal motor patterns during an expiration appears more likely than maintaining separate motor programs to "code" for pause durations observed to be highly variable. Third, the pattern points to flexibility in laryngeal-respiratory coordination, a phenomenon shown to influence the temporal structure of vocal sequences in other mammals. Bats generate two, three, or more echolocation calls (the so-called feeding buzz) per breath as they approach their prey (Smotherman et al., 2006), and most humans are able to realize a sequence of 20 syllables in one breath during a fast speech sequence. Producing several modules during a single breath requires increased control of respiration, but what was thought to be a defining step in the evolution of human speech (MacLarnon and Hewitt, 1999) may instead prove to be a basal mammalian characteristic.

The pattern described in the previous paragraph is preceded by deep breaths that increase the available expiratory lung volume, thus enabling the production of two or more modules during the following expiration. A deep breath ("augmented breath" or "sigh") is a respiratory phenomenon well known in mammals, including rats (Bartlett, 1971; Widdicombe, 2003), and its rate of occurrence has been correlated to emotional state in both rats (Soltysik and Jelen, 2005) and humans (Vlemincx et al., 2011). This study's finding that deep breaths form part of the rat's vocal behavior is remarkable because it may implicate an additional physiological basis for communicating emotional information. Information about a rat's psychological state is relayed through various ultrasonic call types (e.g., Brudzynski, 2013), but it is possible that additional information may be encoded via the respiratory and laryngeal integration reflected in call rates and/or module combination. This also suggests that rats use respiratory modulations in a manner similar to humans (adjusting lung volume before a vocal utterance) with cascading effects on vocal rates, sound pressure level, and fundamental frequency contour (Watson et al., 2003).

Rodents are important models to the evolutionary study of vocal communication (Pollard and Blumstein, 2012). Mammalian vocal behavior is characterized by a large diversity in acoustic features at the species level and above (e.g., Tembrock, 1996), as well as by differences among individuals (the present study). Acoustic diversity can be generated by variation in both vocal morphology and motor patterns driving sound production. The recombination of modules could be a mechanism to increase 
vocal complexity, which itself plays an important role in the evolution of vocal communication (e.g., Bass and McKibben, 2003; Freeberg et al., 2012) either by simply increasing the size of the vocal repertoire or by generating new communicative meaning (e.g., Arnold and Zuberbühler, 2008; ten Cate and Okanoya, 2012). In rats, trills reflect a positive affective state (in contrast to calls with a flat frequency contour: e.g., Burgdorf et al., 2008), but single module trills and composite calls containing a trill unit have been lumped in previous studies. Whether a trill (or any unit) in combination generates different meaning than the same unit uttered singly (i.e., reflecting ambiguity in the sender or amplification of an affective state) remains to be explicitly tested.

\section{References}

Amador A, Perl YS, Mindlin GB, Margoliash D (2013) Elemental gesture dynamics are encoded by song premotor cortical neurons. Nature 495: 59-64. CrossRef Medline

Arnold K, Zuberbühler K (2008) Meaningful call combinations in a nonhuman primate. Curr Biol 18:R202-R203. CrossRef Medline

Arriaga G, Zhou EP, Jarvis ED (2012) Of mice, birds, and men: the mouse ultrasonic song system has some features similar to humans and song learning birds. PLoS One 7:e46610. CrossRef Medline

Bartlett D Jr (1971) Origin and regulation of spontaneous deep breaths. Respir Physiol 12:230-238. CrossRef Medline

Bass AH, McKibben JR (2003) Neural mechanisms and behaviors of acoustic communication in teleost fish. Prog Neurobiol 69:1-26. CrossRef Medline

Benjamini Y, Hochberg Y (1995) Controlling the false discovery rate: a practical and powerful approach to multiple testing. J R Statist Soc B 57:289-300.

Bizzi E, Cheung VC, d'Avella A, Saltiel P, Tresch M (2008) Combining modules for movement. Brain Res Rev 57:125-133. CrossRef Medline

Brenowitz EA, Arnold AP (1986) Interspecific comparisons of the size of neural song control regions and song complexity in duetting birds: evolutionary implications. J Neurosci 6:2875-2879. Medline

Browman CP, Goldstein L (1989) Articulatory gestures as phonological units. Phonology 6:201-251. CrossRef

Brudzynski SM (2013) Ethotransmission: communication of emotional states through ultrasonic vocalization in rats. Curr Opin Neurobiol 23: 310-317. CrossRef Medline

Burgdorf J, Kroes RA, Moskal JR, Pfaus JG, Brudzynski SM, Panksepp J (2008) Ultrasonic vocalizations of rats (Rattus norvegicus) during mating, play, and aggression: behavioral concomitants, relationship to reward, and self-administration of playback. J Comp Psychol 122:357-367. CrossRef Medline

Flash T, Hochner B (2005) Motor primitives in vertebrates and invertebrates. Curr Opin Neurobiol 15:660-666. CrossRef Medline

Freeberg TM, Ord TJ, Dunbar RI (2012) The social network and communicative complexity: preface to theme issue. Philos Trans R Soc Lond B Biol Sci 367:1782-1784. CrossRef Medline

Hammerschmidt K, Reisinger E, Westekemper K, Ehrenreich L, Strenzke N, Fischer J (2012) Mice do not require auditory input for the normal development of their ultrasonic vocalizations. BMC Neurosci 13:40. CrossRef Medline

Hickok G (2012) Computational neuroanatomy of speech production. Nat Rev Neurosci 13:135-145. CrossRef Medline

Holy TE, Guo Z (2005) Ultrasonic songs of male mice. PLoS Biol 3:e386. CrossRef Medline

Jürgens U (2002) Neural pathways underlying vocal control. Neurosci Biobehav Rev 26:235-258. CrossRef Medline

Jürgens U, Hage SR (2007) On the role of the reticular formation in vocal pattern generation. Behav Brain Res 182:308-314. CrossRef Medline

Kent RD, Kent JF, Weismer G, Duffy JR (2000) What dysarthrias can tell us about the neural control of speech. J Phon 28:273-302. CrossRef

Kikusui T, Nakanishi K, Nakagawa R, Nagasawa M, Mogi K, Okanoya K
(2011) Cross fostering experiments suggest that mice songs are innate. PLoS One 6:e17721. CrossRef Medline

Kobayasi KI, Hage SR, Berquist S, Feng J, Zhang S, Metzner W (2012) Behavioural and neurobiological implications of linear and non-linear features in larynx phonations of horseshoe bats. Nat Commun 3:1187. CrossRef Medline

MacLarnon AM, Hewitt GP (1999) The evolution of human speech: the role of enhanced breathing control. Am J Phys Anthropol 109:341-363. CrossRef Medline

MacNeilage PF (1970) Motor control of serial ordering of speech. Psychol Rev 77:182-196. CrossRef Medline

Mahrt EJ, Perkel DJ, Tong L, Rubel EW, Portfors CV (2013) Engineered deafness reveals that mouse courtship vocalizations do not require auditory experience. J Neurosci 33:5573-5583. CrossRef Medline

Miller CT, Flusberg S, Hauser MD (2003) Interruptibility of long call production in tamarins: implications for vocal control. J Exp Biol 206:2629 2639. CrossRef Medline

Moore JM, Székely T, Büki J, Devoogd TJ (2011) Motor pathway convergence predicts syllable repertoire size in oscine birds. Proc Natl Acad Sci U S A 108:16440-16445. CrossRef Medline

Perkell JS (2012) Movement goals and feedback and feedforward control mechanisms in speech production. J Neurolinguistics 25:382-407. CrossRef Medline

Pollard KA, Blumstein DT (2012) Evolving communicative complexity: insights from rodents and beyond. Philos Trans R Soc Lond B Biol Sci 367:1869-1878. CrossRef Medline

Riede T (2011) Subglottal pressure, tracheal airflow and intrinsic laryngeal muscle activity during rat ultrasound vocalization. J Neurophysiol 106: 2580-2592. CrossRef Medline

Riede T (2013) Call type specific motor patterns in rat ultrasound vocalization. J Exp Zool A Ecol Genet Physiol 319:213-224.

Robinson JG (1984) Syntactic structures in the vocalizations of wedgecapped capuchin monkeys, Cebus olivaceus. Behaviour 90:46-79. CrossRef

Scattoni ML, Gandhy SU, Ricceri L, Crawley JN (2008) Unusual repertoire of vocalizations in the BTBR $\mathrm{T}+\mathrm{tf} / \mathrm{J}$ mouse model of autism. PLoS One 3:e3067. CrossRef Medline

Smotherman MS (2007) Sensory feedback control of mammalian vocalizations. Behav Brain Res 182:315-326. CrossRef Medline

Smotherman M, Kobayasi K, Ma J, Zhang S, Metzner W (2006) A mechanism for vocal-respiratory coupling in the mammalian parabrachial nucleus. J Neurosci 26:4860-4869. CrossRef Medline

Soltysik S, Jelen P (2005) In rats, sighs correlate with relief. Physiol Behav 85:598-602. CrossRef Medline

Tembrock G (1996) Akustische Kommunikation der Säugetiere. Darmstadt: Wissenschaftl Buchgesell.

ten Cate C, Okanoya K (2012) Revisiting the syntactic abilities of nonhuman animals: natural vocalizations and artificial grammar learning. Philos Trans R Soc Lond B Biol Sci 367:1984-1994. CrossRef Medline

Vlemincx E, Taelman J, De Peuter S, Van Diest I, Van den Bergh O (2011) Sigh rate and respiratory variability during mental load and sustained attention. Psychophysiology 48:117-120. CrossRef Medline

Watson PJ, Ciccia AH, Weismer G (2003) The relation of lung volume initiation to selected acoustic properties of speech. J Acoust Soc Am 113: 2812-2819. CrossRef Medline

Widdicombe J (2003) Functional morphology and physiology of pulmonary rapidly adapting receptors (RARs). Anat Rec A Discov Mol Cell Evol Biol 270:2-10. CrossRef Medline

Wöhr M, Schwarting RK (2012) Testing social acoustic memory in rats: effects of stimulus configuration and long-term memory on the induction of social approach behavior by appetitive $50-\mathrm{kHz}$ ultrasonic vocalizations. Neurobiol Learn Mem 98:154-164. CrossRef Medline

Wright JM, Gourdon JC, Clarke PB (2010) Identification of multiple call categories within the rich repertoire of adult rat $50-\mathrm{kHz}$ ultrasonic vocalizations: effects of amphetamine and social context. Psychopharmacology 211:1-13. CrossRef Medline 